\title{
Acute alcohol intoxication impairs segmental body alignment in upright standing
}

\section{Anna Hafstrom ${ }^{\mathrm{a} *}$, Mitesh Patel $^{1,2}$, Fredrik Modig ${ }^{1}$, Mans Magnusson ${ }^{1}$ and Per- Anders Fransson ${ }^{1}$}

A Department of Otorhinolaryngology, Head and Neck Surgery, Clinical Sciences, Lund University, Lund, Sweden

B Department of Clinical Neuroscience, Neuro-Otology Department, Imperial College, London, UK

*Corresponding author: A. Hafstrom, Department of Otorhinolaryngology

Head and Neck Surgery, Clinical Sciences, Lund University,

S-221 85 Lund, Sweden. Tel.: +46 171 770; Fax: +46 211

0968; E-mail: anna.hafstrom@skane.se.

\begin{abstract}
Balance control when standing upright is a complex process requiring input from several partly independent mechanisms such as coordination, feedback and feedforward control, and adaptation. Acute alcohol intoxication from ethanol is recognized as a major contributor to accidental falls requiring medical care. This study aimed to investigate if intoxication at 0.06 and $0.10 \%$ blood alcohol concentration affected body alignment. Mean angular positions of the head, shoulder, hip, and knee were measured with 3D-motion analysis and compared with the ankle position in 25 healthy adults during standing with or without perturbations, and with eyes open or closed. Alcohol intoxication had significant effects on body alignment during perturbed and unperturbed stance, and on adaptation to perturbations. It induced a significantly more posterior alignment of the knees and shoulders, and a tendency for a more posterior and left deviated head alignment in perturbed stance than when sober. The impact of alcohol intoxication was most apparent on the knee alignment, where availability of visual information deteriorated the adaptation to perturbations. Thus, acute alcohol intoxication resulted in inadequate balance control strategies with increased postural rigidity and impaired adaptation to perturbations. These factors probably contribute to the increased risk of falling when intoxicated with alcohol.
\end{abstract}

Keywords: Ethanol, balance, postural control, vision, adaptation

\section{Introduction}

Acute alcohol intoxication from ethanol consumption is recognized as a major contributor to many accidental falls causing traumatic injuries, including brain injury, where the severity of the injuries 
has been found to correlate directly with Blood Alcohol Concentration (BAC) [16,30]. Maintaining an accurate body alignment with respect to gravity, support surfaces, the visual surround, internal references and external perturbations is one of the key goals in postural control. Standing upright is dependent on the CNS integrating, interpreting, and processing input from vestibular, visual, and somatosensory information and the accurate coordination of movement response strategies to stabilize body alignment during both self-initiated and external perturbations [12]. The specific response strategy selected depends not only on the characteristics of the external postural displacement but also on the individual's expectations, goals and prior experience and cognitive function.

The least energy consuming and most common movement pattern to maintain postural stability in unperturbed and perturbed standing is the usually called the single-link pattern or ankle strategy. Similar movements of all body segments with corrective movements introduced around the ankle joints identify this strategy [9]. When balance control is more challenged this simplified movement strategy is replaced by a multisegmented movement pattern with corrective movements about other joints, such as at the knees or hips [13]. A general strategy used when submitted to substantial balance perturbations, such as vibration perturbations against the calf muscles or when closing the eyes, is to change the positions of the head, shoulders, hip and knees more forward relative to the ankle joint, i.e., to use a new posture that causes realignment of all body segments $[9,25]$.

Acute alcohol intoxication affects balance control and the vestibuloocular system in numerous ways $[2,5,8,17,27,32]$. Earlier studies have shown that alcohol intoxication from about $0.07 \%$ BAC compromises unperturbed standing balance $[23,31]$, whereas perturbed standing balance can be affected from about $0.06 \%$ BAC $[20,21,26,33]$.

We have earlier demonstrated that increasing alcohol intoxication increases the visual field dependence and impairs the perception of the true visual vertical and horizontal [11], that vision provides a weaker contribution to postural control during alcohol intoxication [22], and that alcohol intoxication causes increased linear movements of different body segments [20] where knee movements is less correlated with movements of other body parts when visual information is unavailable [26]. Our present hypothesis was that alcohol intoxication would also cause misalignment of the head and other body segments during upright standing. The aim was to study the alignment of different body segments at different blood alcohol concentration levels $(0.06 \%$ and $0.10 \% \mathrm{BAC}$ ) with kinematic analysis of the head, shoulder, hip, and knee positions in unperturbed and perturbed stance. Furthermore, we wanted to investigate whether alcohol intoxication affected the ability for adaptation and maintaining accurate body alignment during repeated balance perturbations by calf muscle vibration.

\section{Methods}

This report is part of a larger study concerning the effects of alcohol on the vestibular, oculo-motor, and postural systems $[10,11,20-22,26]$. The test subjects were thoroughly examined with vestibular head impulse tests, headshake and positional nystagmus tests, subjective visual horizontal and vertical tests (SVHV), eye pursuit movements, hearing and visual acuity tests. They were excluded 
from having a medical or vestibular history of previous vertigo, balance problems, inner ear disease, earlier bacterial meningitis, major CNS-trauma, cardiovascular disease or serious injuries involving their lower extremities and excessive alcohol consumption that could possibly cause balance deficits. Thus the final study group consisted of 25 healthy participants ( 13 women and 12 men, mean age 25 years) after having excluded two subjects; one for not reaching intended BAC and one due to failing the SVH-V test [11]. The study design was approved by the Ethics Committee of the Medical Faculty, Lund University, Lund, Sweden and was performed in accordance with the latest version of the Helsinki declaration. Written informed consent was obtained from all participants.

Tests were performed once a week for three consecutive weeks at three different blood alcohol concentrations (BAC): $0.0 \%, 0.06 \%$, and $0.10 \%$, in a randomized order as described elsewhere and recently published $[11,21,22,26]$. Thus, the amount of $70 \%$ ethanol diluted in elderflower juice depended on gender and weight. After consuming the drink, alcohol concentration was measured every 15 minutes by an Evidenzer breath analyzer (Nanopuls AB, Uppsala, Sweden) until a plateau phase could be identified in the BAC recordings and tests were performed in the descending phase of BACs [26].

Subjects stood in a relaxed posture on a platform during quiet standing and pseudorandom pulses of calf muscle vibration with eyes closed or open as described elsewhere $[9,15]$. Five markers were placed on anatomical landmarks on the right side of the subjects; the 'head' marker was attached to os zygomaticum, the 'Shoulder' marker to tuberculum majus, the 'hip' marker to the crista iliaca, the 'knee' marker to the lateral epicondyle of femur, and the 'ankle' marker to the lateral distal fibula head. Angular positions of the different markers were recorded in anteroposterior, lateral, and vertical directions with a resolution of $0.4 \mathrm{~mm}$ with the ultrasound ZebrisTM3D-Motion Tracking system [26]. The mean angular positions were calculated from the respective marker's height and anteroposterior or lateral position using the ankle marker as the zero-position reference with an error of less than $1.5 \%[9]$.

Group mean values for all the marked body segments were obtained for five periods for each trial condition: an unperturbed stance period ( $0-30 \mathrm{~s}$ ), and from four 50-second periods of vibration (Period 1: 30-80 s; Period 2: 80-130 s; Period 3: 130-180 s; Period 4: 180-230 s). Higher positive values indicated a more forward position in the anteroposterior direction and deviation more to the right in the lateral direction. The combined information from the all the markers illustrate multisegmental alignment of the body during respective test conditions and thus, whether body alignment is changed to meet the plausible increased challenges due to perturbations and alcohol intoxication.

Since the main objective of this study was to describe the effects of alcohol intoxication on the body alignment, the ankle was used as zero point reference for the angle calculation. Each angle value represents one segment in the multi-segmental chain forming the body alignment. Hence, each angle presented illustrates how the positions and angles at each individual joint contribute to the body leaning and how the total alignment is altered from local changes, and thus the potential risk of falling from a realignment change. 


\section{Statistical analysis}

A multifactorial statistical method in two steps was used to analyze the data. First, a multifactorial univariate General Linear Model analysis of variance (GLM ANOVA) evaluation of the average angular positions in the anteroposterior and lateral directions was performed.

The effects of alcohol intoxication ('Alcohol': $0.0 \%, 0.06 \%$ or $0.10 \%$ BAC; degrees of freedom (d.f.) 2), availability of visual information ('Vision': eyes closed or eyes open; d.f. 1), direction of recorded angular position ('Direction': anteroposterior or lateral; d.f. 1), and when applicable the period of vibration ('Period': periods 1-4; d.f. 3) and their interactions on the average angular position values during quiet stance and during balance perturbations were analyzed. The interactions reveal whether certain combinations of main factor conditions might influence the outcome. The Wilcoxon matched-pairs signed-rank test was used for the post hoc pair-wise analysis. Nonparametric statistical tests were used in the post hoc evaluation because this data was not normally distributed. The Bonferroni procedure was applied to pair wise comparisons when appropriate. In all other analysis, $p$-values $<0.05$ were considered statistically significant [1].

\section{Results}

As seen in Table 1, alcohol intoxication significantly affected the average angular position of the head (both in quiet stance and during balance perturbations) and knees (during balance perturbations) as expressed by the significant 'Alcohol' factor. The availability of visual information had a significant impact on head position both during quiet stance and balance perturbations.

Significant interactions were found for many of the factors. All these interactions included the Direction factor and as expected, the average angular position of all body segments was affected more in the anteroposterior than in the lateral direction as illustrated by the significant 'Direction' factor $(p<0.001)$. Thus, a second GLM ANOVA was performed separately analyzing average angular positions in the anteroposterior and lateral directions (Table 2). No factors had significant interactions according to this analysis.

During balance perturbations, increasing alcohol intoxication significantly affected alignment of the knees $(p<0.001)$ and shoulders $(p=0.008)$ to more posterior positions. Alcohol intoxication significantly affected head alignment in the lateral direction $(p<0.005)$ inducing a more leftward average angular head position. Availability of visual information had a significant impact of the head and shoulder alignment in the anteroposterior direction (Table 2).

\section{Segmental body alignment when sober}

When subjects were sober standing unperturbed, the head position was more anterior when standing with eyes closed (EC) compared to with eyes open (EO) $(p=0.032)$ (Fig. 1). The onset of perturbations in Period 1 induced more anterior positions of the head (EO and EC), knees (EO), and shoulders (EO) $(p<0.050)$. The more anterior head, shoulder and knee positions were significantly increased as perturbations continued $(p<0.001)$. With EC the changes in knee and shoulder positions by the perturbations were less prominent and came later. The perturbations induced 
smaller changes in the hip position. In the lateral direction no significant changes in body alignment were seen when sober (Fig. 1E).

\section{Segmental body alignment when intoxicated}

As seen in Fig. 1D, alcohol intoxication affected the average angular position of the knees, both with $\mathrm{EO}$ and EC. The change to a more posterior knee alignment was most pronounced during perturbations. During perturbations with EO, the knee position was significantly more posterior when intoxicated compared to being sober, both at $0.06 \%(p<0.01)$ and $0.10 \%$ BAC $(p<0.0167)$. With EC and $0.06 \% \mathrm{BAC}$, the knee position was significantly more posterior at the onset of perturbations than when being sober (Period $1 p=0.014$ ), but less so as the perturbations continued (Period 2 and $3 p<0.026)$. In quiet stance the knees also had tendency $(p<0.05)$ for a more posterior knee position than when sober, both with EO and EC.

Alcohol intoxication significantly affected alignment of the shoulders (Table 2), but not the average angular positions of the hips. With EO the shoulders had a tendency for a more posterior position at $0.10 \%$ BAC than when sober in perturbation period $2(p<0.05)$. Alcohol intoxication affected the average angular position for the head as indicated by 'Alcohol' in the GLM ANOVA ( $p=0.019)$ (Table 1). However, the alignment changes were more significant in the lateral than in the anteroposterior direction according to Table $2(p=0.005)$. A tendency for a leftward deviation of head alignment was found with increasing alcohol intoxication (Fig. 1E). The more leftward head position when intoxicated was more evident during perturbations with EC (Period $3 p=0.013$ ). According to the post Hoc analysis alcohol intoxication had significant effect on the position of the head in the anteroposterior direction, especially with EO (Fig. 1A). During perturbations and EC, no significant differences were found for the head alignment in the anteroposterior direction with increasing alcohol levels.

\section{Effect of alcohol intoxication on adaptation to perturbations}

With repeated balance perturbations, the position of the head, shoulders and hips (but not knees) was influenced over time as indicated by 'Period' in Tables 1 and 2. When subjects were intoxicated, just like when they were sober, the initial 50 seconds of vibratory perturbations induced a more forward inclination and they leaned even more forward as the perturbations continued. However, as seen in Fig. 1, when intoxicated there was a difference in adaptation to the perturbations depending if eyes were open or closed. When intoxicated being perturbed with EO, the average angular position of the knees (and head) never reached the same anterior position as when sober.

\section{Discussion}

An adequate strategy enhancing stability when submitted to substantial balance perturbations is to use an increased forward inclination and a more flexible posture $[9,25,28]$. The present results corroborates that, when sober and submitted to substantial balance perturbations and/or when visual information is not available, a new posture with a more forward position of the head, shoulders, hip and knees relative to the ankle joint takes place, i.e. a realignment of all body 
segments occurs. When intoxicated, however, a significant more posterior alignment of the knees was found. Furthermore, a tendency for a more posterior and left deviated head position and a more posterior shoulder position was noted.

The impact of alcohol intoxication with a more posterior and rigid alignment of body segments was more apparent when visual information was available than when not. These results are in line with the earlier findings of deteriorated ability to maintain balance during eyes-open when intoxicated, revealing an inadequate compensation of the ethanol-induced ataxia by visual stabilization $[23,24]$. The current findings also concur with the findings of Modig et al. who concluded that mechanoreceptive sensation is of increased importance for human postural control under alcohol intoxication since the ability to handle balance perturbations when intoxicated is strongly dependent on accurate mechanoreceptive sensation and efficient reweighting [22]. With eyes closed, a reweighting to mechanoreceptors in the foot soles should already have occurred and thus faster adaptation to the random balance perturbations (vibration towards the calf muscles) is to be expected even when intoxicated. The present findings of more prominent changes in balance control strategy when intoxicated and visual information is available (than when not) contradicts some earlier studies concerning the effects of ethanol on postural stability. However, in these studies dynamic or static posturography tests were used and also much higher blood alcohol levels than in the present study $[6,19]$.

The change in the verticality of the head position, exhibited by a more posterior and left deviated head position with increasing alcohol intoxication, could suggest impairment in vertical positioning of the head which might be caused by an impairment in the perception of the true vertical and horizontal. This reasoning is supported by the findings that the alcohol-related changes of the head position were more obvious with EC than with EO in the lateral direction. The representation of the perceived visual vertical is based on the integration, interpretation, and processing of vestibular, visual and somatosensory (particularly cervical) in put by the CNS. By testing the perceived visual vertical and horizontal, it is possible to study the role of gravitational vestibular cues and indeed, when studying the same subjects, Hafstrom et al. recognized that alcohol intoxication caused increased deviations of the subjective visual vertical and horizontal to the left [11]. Thus, the present findings corroborate that the visual system is particularly affected by alcohol intoxication and plays a major role in maintaining postural stability.

The findings of a more posterior position of the knees during vibration perturbations when intoxicated, foremost when visual information was available but also when not, suggests that compared to being sober, a different body alignment strategy had to be deployed in order to compensate for the destabilizing effects of alcohol. The positioning of more posterior and thus a more rigid alignment of the knee joints with increasing alcohol intoxication might imply reduced possibilities for dorsiflexion at the ankles to compensate for the perturbations since random vibration against the calf generate body movement primarily in an anterior-posterior direction [7]. Thus, reduced knee movements due to knee rigidity should contribute to sagittal plane postural instability. Interestingly, increased knee rigidity has been observed in patients with spino-cerebellar ataxia [18] and several studies have reported that acute alcohol intoxication appears to cause a cerebellar deficit [6,23], possibly by blocking cerebellar parallel-fibre long term-depression [4]. Patel et al. looked at the segmental movement pattern for the same subjects as in the present study and showed that when balance was repeatedly perturbed, the normal knee movements became 
significantly less correlated to other body movements and more independent over time [26]. The more independent knee movements were present when visual information was unavailable and the results were interpreted as alcohol intoxication enforcing the CNS to use a more precautious segmental body movement coordination pattern. One can hypothesize that increased knee movements and a more anterior knee alignment with EC compared to EO while intoxicated, is part of a proficient CNS-strategy to avoid falling. Furthermore, a more anterior knee position ought to be a biomechanical advantage for both the ankle and knee strategy when greater movement around the ankle joint is needed.

In healthy sober subjects repeated balance perturbations usually result in sensorimotor adaptation (motor learning), which may cause sensory re-weighting and altered feedback and feedforward motor control responses [3]. The present results indicate that despite alcohol intoxication an adaptation of body segment alignment to the perturbations was present mimicking sober behavior, with the exception for the knee (and head) alignment with eyes open. With repeated balance perturbations the position of the knees never reached the same more forward position they had when sober. Thus, when intoxicated and visual information was available, impaired adaptation to the perturbations was seen in the anteroposterior direction for knee alignment. With eyes closed, however, there was a delay in the adaptation to the perturbations with a more forward of alignment of the knees. Modig et al investigated the destabilization rates of linear movement variance for the same body segments over time and found an apparent decrease in the adaptation efficiency with increasing BAC in anteroposterior direction [20]. Furthermore, the delay or absence of knee alignment adaptation to the perturbations when intoxicated corroborates earlier findings that alcohol intoxication significantly delay balance responses, thus making balance control difficult when intoxicated $[23,33]$. Thus, when the human CNS is submitted to alcohol intoxication, it is apparent that a vast array of functions related to postural control is affected. In an attempt to rate the relative importance of the functional changes we have investigated so far in the perspective of increasing the fall risk $[10,11,20-22,26]$, in our opinion, two changes in postural control due to alcohol intoxication pose the largest risks for falls.

The first change is the abrupt decline in the ability to effectively use certain kinds of sensory information. Particularly visual input, possibly in conjunction with disrupted vestibular information, seems to lose substantial capacity to provide reliable postural feedback information, conceivably because alcohol intoxication distorts the oculomotor functions [10]. A key manifestation of losing the sensory robustness of visual information, when intoxicated, is the decreased lateral stability over time to the level where the stability with eyes open matches the one with eyes closed after 4 minutes [21]. Another manifestation of changes in the sensory information reliability was the increasingly larger importance of mechanoreceptive information from the feet over time at high BAC levels [22].

The second change is the general decline in stability observed over time. Our hypothesis is that attentiveness and heightened awareness to compensate for perturbations may have a substantial beneficial effect to enhance the balance performance and to some extent suppress the effects of alcohol intoxication. However, as shown in an earlier study by Modig et al., the initially elevated attention when submitted to perturbations dropped after about a minute and the relative stability rapidly declined. After 4 minutes the postural performance was several hundred percent worse than during unperturbed conditions, particularly in the lateral direction [20]. Hence, the largest risk for 
falls from alcohol intoxication, poses, in our opinion, the gradual subtle functional declines that reaches full effects first when the subjects' attention to controlling the body's' stability have subsided.

As shown in the present study, the control of posture alignment appears to be less affected by alcohol intoxication and thus one of the more robust functions in postural control. The body alignment seemingly never changed abruptly, even at $0.1 \%$ BAC or when submitted to the initial balance perturbations that evoked large body movements over large movement spans. Instead, in our assessments, the alignment control acted with strategic rationale making changes that given the circumstances enhanced the stability, though the implementations of these changes were slower while intoxicated. When presented in degrees, the changes in body alignments and movement spans rarely exceeded 2 degrees, which may appear as changes of minor clinical significance. However, these changes should be considered in relation to the normal biomechanical stability ranges in upright stance. When presented as the anteroposterior angle between the ankle joint position and the center of mass position, the stability limit for leaning is estimated to be about 4-5 degrees backward and 12 degrees forward [14,29]. Hence, a body posture realignment or increased movement span of a few degrees may imply that proportionally large parts of the remaining stability marginal have been exploited, and thus, the risk for falling is substantially higher.

The current findings of more posterior alignment of the knees and head during unperturbed stance (but more prominent during perturbed stance) indicates that measuring body alignment is a sensitive method to detect the effects of alcohol intoxication on postural control. When investigating the same subjects as in the present study, the effects of alcohol intoxication on movement coordination patterns of different body segments were negligible in unperturbed standing [26].

\section{Conclusions}

This study uncovered that alcohol intoxication from ethanol has significant effects on segmental body alignment and adaptation to balance perturbations. The effects of alcohol intoxication at 0.06 and $0.10 \%$ BAC were more evident during balance perturbations than in quiet standing. The more posterior and rigid knee positioning and the tendency for a more posterior and left deviated head position with increasing alcohol intoxication may be contributing factors to fall trauma in addition to the direct effect of alcohol intoxication. Furthermore, the changes in body alignment in the anteroposterior direction were more pronounced when visual information was available compared to when it was not, indicating an increased visual dependency with increasing levels of alcohol intoxication. Thus, acute al cohol intoxication resulted in inadequate balance control strategies with increased postural rigidity and impaired adaptation to perturbations. These factors probably contribute to the increased risk of falling when intoxicated with alcohol.

\section{References}

[1] D.G. Altman, Practical statistics for medical research, Chapman \& Hall, London, 1991. 
[2] R.W. Baloh, S. Sharma, H. Moskowitz and R. Griffith, Effect of alcohol and marijuana on eye movements, Aviat Space Environ Med 50 (1979), 18-23.

[3] A.J. Bastian, Learning to predict the future: The cerebellum adapts feedforward movement control, Curr Opin Neurobiol 16 (2006), 645-649.

[4] A. Belmeguenai, P. Botta, J.T. Weber, M. Carta, M. De Ruiter, C.I. De Zeeuw, C.F. Valenzuela and C. Hansel, Alcohol impairs long-term depression at the cerebellar parallel fiberPurkinje cell synapse, J Neurophysiol 100 (2008), 3167-3174.

[5] A. Berthoz, L. Young and F. Oliveras, Action of alcohol on vestibular compensation and habituation in the cat, Acta Otolaryngol 84 (1977), 317-327.

[6] H.C. Diener, J. Dichgans, M. Bacher, J. Hulser and H. Liebich, Mechanisms of postural ataxia after intake of alcohol, Z Rechtsmed 90 (1983), 159-165.

[7] G. Eklund, Further studies of vibration-induced effects on balance, Ups J Med Sci 78 (1973), 6572.

[8] M. Fetter, T. Haslwanter, M. Bork and J. Dichgans, New insights into positional alcohol nystagmus using threedimensional eye-movement analysis, Ann Neurol 45 (1999), 216-223.

[9] P.A. Fransson, M. Hjerpe and R. Johansson, Adaptation of multi-segmented body movements during vibratory proprioceptive and galvanic vestibular stimulation, J Vestib Res 17 (2007), 47-62.

[10] P.A. Fransson, F. Modig, M. Patel, S. Gomez and M. Magnusson, Oculomotor deficits caused by $0.06 \%$ and $0.10 \%$ blood alcohol concentrations and relationship to subjective perception of drunkenness, Clin Neurophysiol 121 (2010), 2134- 2142.

[11] A. Hafstrom, F. Modig, M. Karlberg and P.A. Fransson, Increased visual dependence and otolith dysfunction with alcohol intoxication, Neuroreport 18 (2007), 391-394.

[12] F.B. Horak, Postural orientation and equilibrium: What do we need to know about neural control of balance to prevent falls? Age Ageing 35 Suppl 2 (2006), ii7-ii11.

[13] F.B. Horak and L.M. Nashner, Central programming of postural movements: Adaptation to altered support-surface con- figurations, J Neurophysiol 55 (1986), 1369-1381.

[14] F.B. Horak, C.L. Shupert and A. Mirka, Components of postural dyscontrol in the elderly: A review, Neurobiol Aging 10 (1989), 727-738.

[15] R. Johansson, System Modeling and Identification, in, Prentice Hall, Englewood Cliffs. NJ, 1993, p. 221.

[16] J.J. Johnston and S.J. McGovern, Alcohol related falls: An interesting pattern of injuries, Emerg Med J 21 (2004), 185- 188.

[17] T. Kubo, Y. Sakata, A. Koshimune, S. Sakai, K. Ameno and I. Ijiri, Positional nystagmus and body sway after alcohol ingestion, Am J Otolaryngol 11 (1990), 416-419. 
[18] U.M. Kung, C.G. Horlings, F. Honegger, H.P. Kremer, B.R. Bloem, B.P. van De Warrenburg and J.H. Allum, Postural instability in cerebellar ataxia: Correlations of knee, arm and trunk movements to center of mass velocity, Neuroscience 159 (2009), 390-404.

[19] T. Ledin and L.M. Odkvist, Effect of alcohol measured by dynamic posturography, Acta Otolaryngol Suppl 481 (1991), 576-581.

[20] F. Modig, P.A. Fransson, M. Magnusson and M. Patel, Blood alcohol concentration at 0.06 and $0.10 \%$ causes a complex multifaceted deterioration of body movement control, Alcohol 46 (2012), $75-88$.

[21] F. Modig, M. Patel, M. Magnusson and P.A. Fransson, Study I: Effects of $0.06 \%$ and $0.10 \%$ blood alcohol concentration on human postural control, Gait Posture 35 (2012), 410-418.

[22] F. Modig, M. Patel, M. Magnusson and P.A. Fransson, Study II: Mechanoreceptive sensation is of increased importance for human postural control under alcohol intoxication, Gait Posture 35 (2012), 419-427.

[23] M. Nieschalk, C. Ortmann, A. West, F. Schmal, W. Stoll and G. Fechner, Effects of alcohol on body-sway patterns in human subjects, Int J Legal Med 112 (1999), 253-260.

[24] H.G. Palm, O. Waitz, J. Strobel, J. Metrikat, B. Hay and B. Friemert, Effects of low-dose alcohol consumption on postural control with a particular focus on the role of the visual system, Motor Control 14 (2010), 265-276.

[25] M. Patel, S. Gomez, D. Lush and P.A. Fransson, Adaptation and vision change the relationship between muscle activity of the lower limbs and body movement during human balance perturbations, Clin Neurophysiol 120 (2009), 601-609.

[26] M. Patel, F. Modig, M. Magnusson and P.A. Fransson, Alcohol intoxication at 0.06 and $0.10 \%$ blood alcohol concentration changes segmental body movement coordination, Exp Brain Res 202 (2010), 431-443.

[27] R.B. Post, L.A. Lott, J.I. Beede and R.J. Maddock, The effect of alcohol on the vestibulo-ocular reflex and apparent concomitant motion, J Vestib Res 4 (1994), 181-187.

[28] S.N. Robinovitch, F. Feldman, Y. Yang, R. Schonnop, P.M. Leung, T. Sarraf, J. Sims-Gould and M. Loughin, Video capture of the circumstances of falls in elderly people residing in long-term care: An observational study, Lancet 381 (2013), 47-54.

[29] T. Sinha and B.E. Maki, Effect of forward lean on postural ankle dynamics, IEEE Trans Rehabil Eng 4 (1996), 348-359.

[30] P. Talving, D. Plurad, G. Barmparas, J. Dubose, K. Inaba, L. Lam, L. Chan and D. Demetriades, Isolated severe traumatic brain injuries: Association of blood alcohol levels with the severity of injuries and outcomes, J Trauma 68 (2010), 357-362.

[31] H.H. Thyssen, J. Brynskov and E.C. Jansen, Alcohol and postural imbalance. A force plate study, Z Rechtsmed 87 (1981), 257-260. 
[32] H. Tianwu, Y. Watanabe, M. Asai, K. Shimizu, S. Takada and K. Mizukoshi, Effects of alcohol ingestion on vestibular function in postural control, Acta Otolaryngol Suppl 519 (1995), 127-131.

[33] M.H. Woollacott, Effects of ethanol on postural adjustments in humans, Exp Neurol 80 (1983), $55-68$. 
Table 1

Statistical findings from the GLM univariate ANOVA evaluation of average angular positions of the head, shoulders, hips and knees. 'A $\mathrm{x}$ D' denotes interactions between 'Alcohol' $x$ 'Direction'. 'V x D' denotes 'Vision x Direction', and 'D x P' denotes 'Direction' x 'Period' interactions. The interaction combinations not presented in the table were not significant (NS). The values in the top rows for each parameter represent p-values and the values in the second rows represent F-values

\begin{tabular}{lcccc|cccccccc}
\hline $\begin{array}{l}\text { Angular } \\
\text { position }\end{array}$ & \multicolumn{3}{c|}{ Unperturbed } & \multicolumn{6}{c}{ Perturbed stance } \\
\cline { 2 - 12 } & Alcohol & Vision & Direction & VxD & Alcohol & Vision & Direction & Period & AxD & VxD & DxP \\
\cline { 2 - 13 } Head & $\mathbf{0 . 0 2 3}$ & $\mathbf{0 . 0 4 5}$ & $<0.001$ & $\mathbf{0 . 0 1 7}$ & $\mathbf{0 . 0 1 9}$ & $<0.001$ & $<0.001$ & $<0.001$ & NS & $<0.001$ & $<0.001$ \\
& 3.8 & 4.1 & 4388.9 & 5.7 & 4.0 & 24.3 & 17463.9 & 6.9 & 1.2 & 32.3 & 10.1 \\
\hline Shoulder & NS & NS & $<0.001$ & NS & NS & NS & $<0.001$ & $\mathbf{0 . 0 0 2}$ & $\mathbf{0 . 0 0 1}$ & $<0.001$ & $\mathbf{0 . 0 0 1}$ \\
& 1.0 & 0.3 & 155.4 & 2.6 & 1.0 & 1.6 & 192.2 & 5.1 & 7.1 & 12.4 & 5.4 \\
\hline Hp & NS & NS & $<0.001$ & NS & NS & NS & $<0.001$ & 0.050 & NS & NS & NS \\
& 0.12 & 0.06 & 341.16 & 0.70 & 0.03 & 1.55 & 1693.96 & 2.61 & 1.77 & 0.10 & 2.38 \\
\hline Knee & NS & NS & $<0.001$ & NS & $<0.001$ & NS & $<0.001$ & NS & $<0.001$ & NS & NS \\
& 2.2 & 0.0 & 814.3 & 0.2 & 10.2 & 0.7 & 3742.6 & 1.588 & 7.9 & 0.6 & 2.1 \\
\hline
\end{tabular}


Table 2

Statistical findings from the GLM univariate ANOVA evaluation of average angular position in the anteroposterior (AP) or lateral (LAT) directions. The interaction combinations not presented in the table were not significant (NS). The values in the top rows for each parameter represent p-values and the values in the second rows represent F-values

\begin{tabular}{|c|c|c|c|c|c|c|}
\hline \multicolumn{4}{|c|}{ Angular position Unperturbed } & \multicolumn{3}{|c|}{ Perturbed stance } \\
\hline & & Alcohol & Vision & Alcohol & Vision & Period \\
\hline \multirow[t]{4}{*}{ Head } & $\overline{\mathrm{AP}}$ & NS & 0.011 & NS & $<0.001$ & $<0.001$ \\
\hline & & 2.7 & 6.6 & 1.7 & 37.3 & 11.1 \\
\hline & LAT & NS & NS & 0.005 & NS & NS \\
\hline & & 1.1 & 0.1 & 5.3 & 0.6 & 0.4 \\
\hline \multirow{2}{*}{\multicolumn{2}{|c|}{ Shoulder AP }} & NS & NS & 0.008 & 0.004 & $<0.001$ \\
\hline & & 1.4 & 1.8 & 4.9 & 8.6 & 7.9 \\
\hline & \multirow[t]{2}{*}{ LAT } & NS & NS & NS & NS & NS \\
\hline & & 0.2 & 0.8 & 2.4 & 3.8 & 0.0 \\
\hline \multirow[t]{4}{*}{$\overline{\mathrm{Hip}}$} & $\overline{A P}$ & NS & NS & $\overline{N S}$ & NS & 0.021 \\
\hline & & 0.0 & 0.4 & 0.5 & 0.3 & 3.3 \\
\hline & LAT & NS & NS & NS & NS & NS \\
\hline & & 0.4 & 0.4 & 2.3 & 2.6 & 0.0 \\
\hline \multirow[t]{4}{*}{ Knee } & $\mathrm{AP}$ & NS & NS & $<0.001$ & NS & NS \\
\hline & & 2.2 & 0.1 & 10.5 & 0.7 & 2.2 \\
\hline & LAT & NS & NS & NS & NS & NS \\
\hline & & 0.4 & 0.1 & 2.1 & 0.0 & 0.1 \\
\hline
\end{tabular}


A Head AP

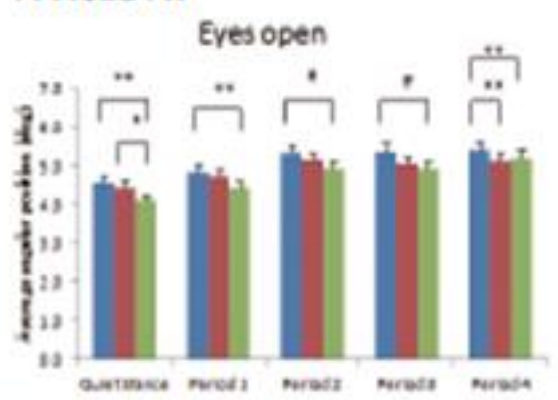

\section{B Shoulders AP}
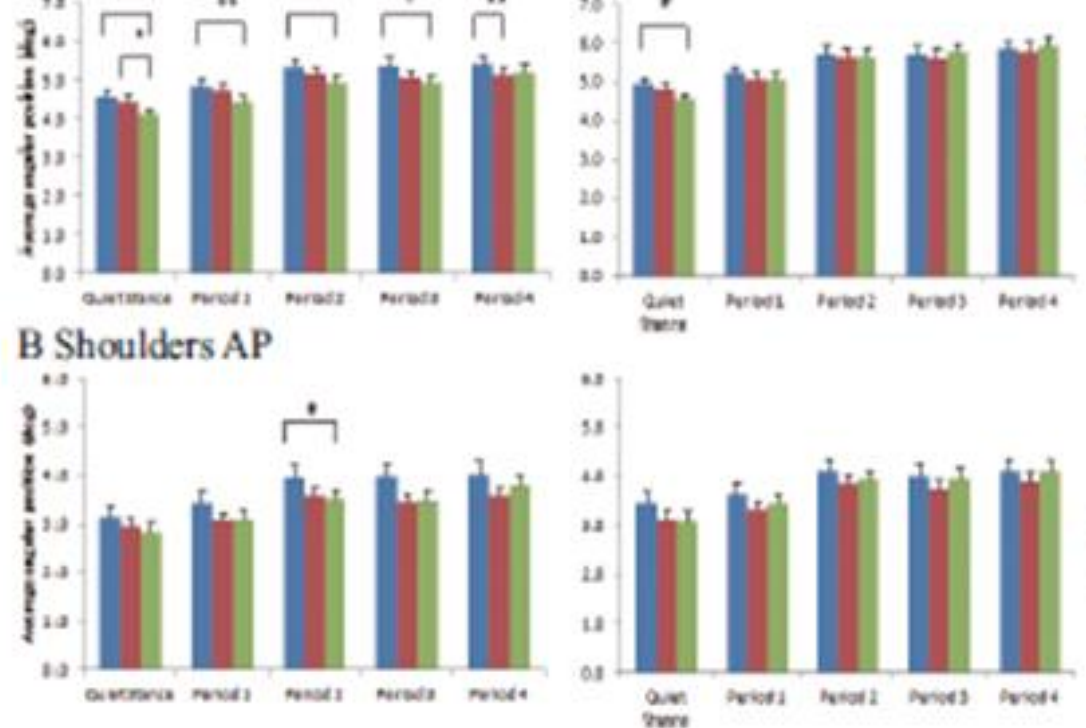

osinse

- sscive

somete

\section{Hips AP}
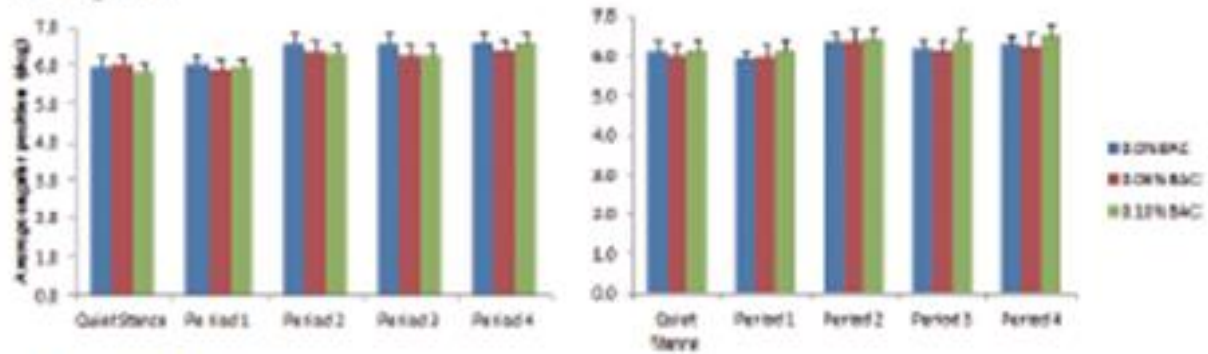

D Knees AP
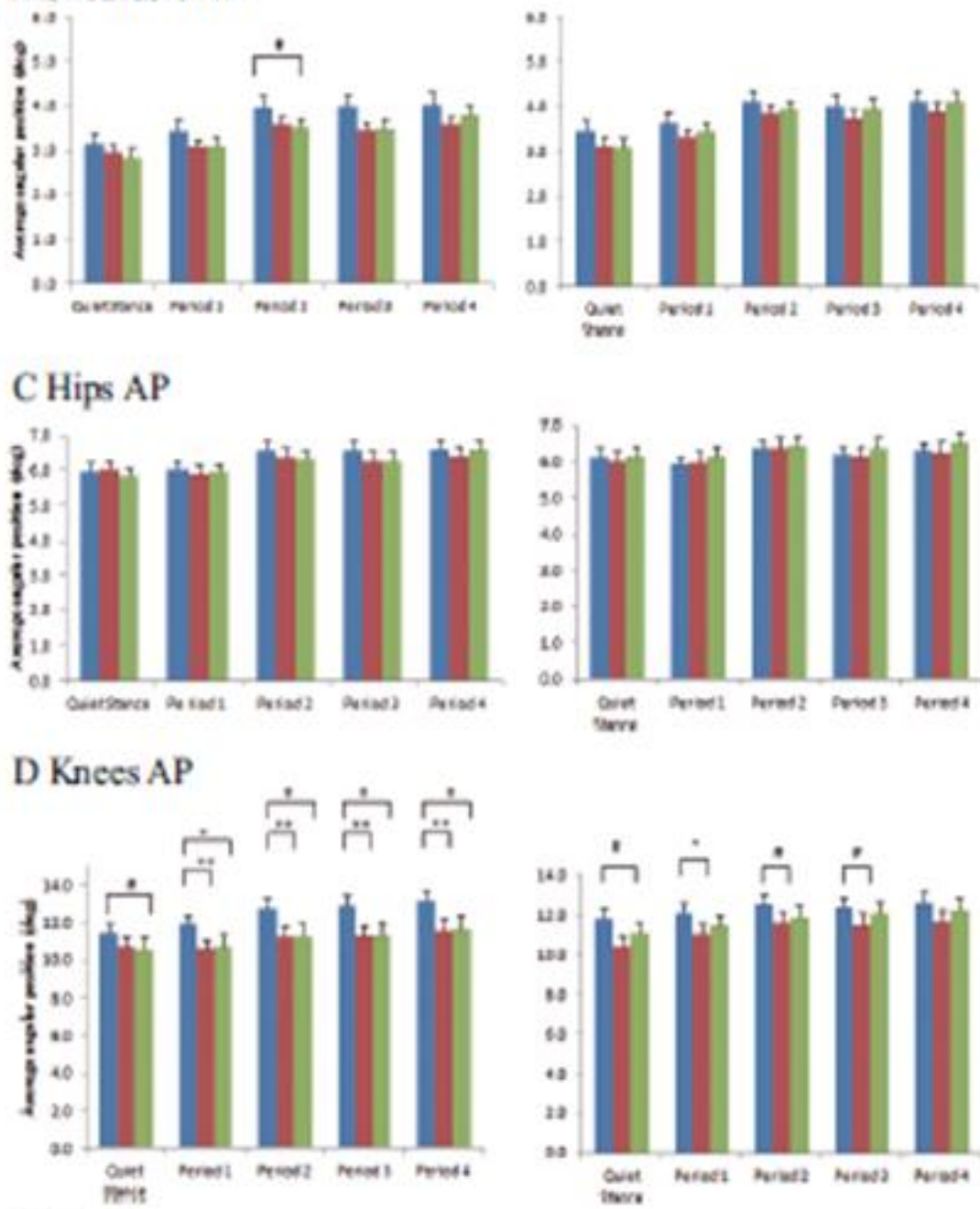

osmac

opsertac

eomear

\section{E Head Lat}

Eves open

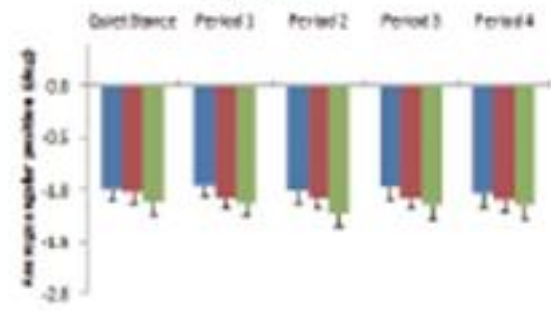

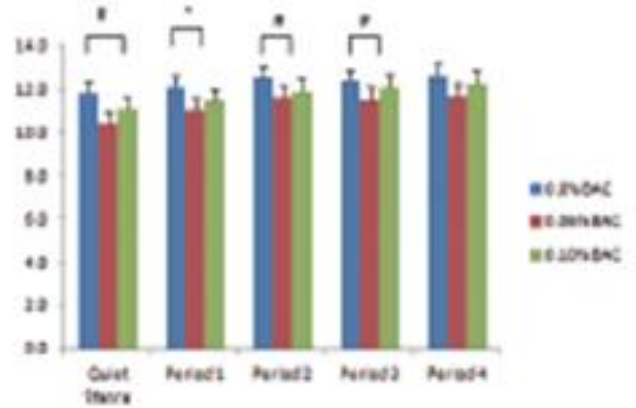

Eyes closed

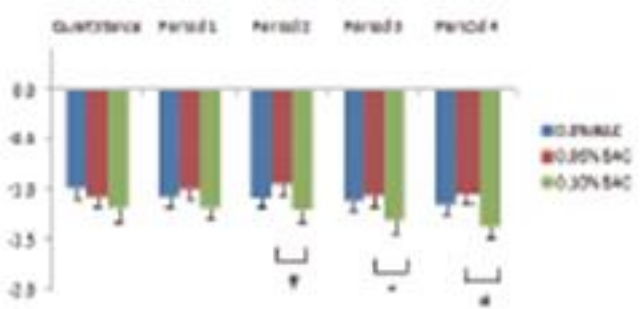

Fig. 1. Noernalized ankropostrioe (AP) average angular positions in degres (mean and SEM) for the Head (1A); Shoulder (1B); Hip (IC); Knee (ID) and in the lateral (L.af) direction for the Head (IE) with cyes open and closed. The figures present the statistical findings made in the post hoc evaluation of the main factor Alcobol. Note that the respective angles are calculated with respect to the "Anikle" marker and, thex, the sizes of the respective angks will be influenced by the respective markers' placement on the body. Furthermort, in the AP dircetion a mote positive value indicates a more antrice position and in the latral dirction a more $x$ gative value indicats a more $k$ ftwand position. $4 d \mathbf{d} n o t=0<0.05$, " denotes $p<0.0167, \cdots$ denotes $p<0.01$ and $\cdots$ denotes $p<0.001$. 